\title{
Silver Nanoparticles by Laser Ablation Confined in Alcohol Using an Argon Gas Environment
}

\author{
David Omar Oseguera Galindo ${ }^{*}$, Oscar Hernández Utrera ${ }^{2}$, Roberto Machorro Mejía ${ }^{3}$, \\ Miguel Ángel Santana Aranda ${ }^{4}$ \\ IPosgrado en Física de Materiales, Centro de Investigación Científica y de Educación Superior de \\ Ensenada, Ensenada, Baja California 22860, México \\ E-mail: davidhor@,cnyn.unam.mx \\ ${ }^{2}$ Catedrático CONACyT, Centro de Nanociencias y Nanotecnología, Universidad Nacional Autónoma de \\ México, Ensenada, Baja California 22860, México \\ ${ }^{3}$ Centro de Nanociencias y Nanotecnología, Universidad Nacional Autónoma de México, Ensenada, Baja \\ California 22860, México \\ ${ }^{4}$ Departamento de Física, Centro Universitario de Ciencias Exactas e Ingenierías, Universidad de \\ Guadalajara, Guadalajara,Jalisco 44430, México
}

The fundamental emission of a Nd:YAG laser $(1064 \mathrm{~nm})$, with an energy $0.1 \mathrm{~J} /$ pulse and a repetition rate of $10 \mathrm{~Hz}$ was employed to synthesize silver nanoparticles by ablation method confined in methanol and ethanol. The experimental setup allows synthesizing materials immersed in flammable solvents using an Argon atmosphere to prevent combustion, making the process safer when high fluence and high repetition rates of laser pulses are used. Transmission electron micrographs were taken for methanol and ethanol samples. The analysis of the methanol sample the day of synthesis and one month later, show that nanoparticles obtained were more dispersed, corresponding to better suspension stability. On the contrary, the images of nanoparticles in ethanol show a strong agglomeration, resulting in a fast sedimentation and less stability.

DOI: $10.2961 /$ jlmn.2016.02.0004

Keywords: nanoparticles, laser ablation, flammable solvents, Argon gas, experimental

\section{Introduction}

At nanometric scales, silver particles exhibit very interesting optical and electronic properties that make them suitable for a large range of applications going from bactericidal agent, biological sensor, to catalysis [1-5] or optical data storage [6]. One of the main characteristics of silver nanoparticles is an absorption peak around $400 \mathrm{~nm}$, which is extensively related to the superficial plasmon resonance, where the peak position depends on the refractive index of the medium and the particle size [7]. Additionally, in case of having a dielectric shield, the peak position would also depend on the shell thickness and its dielectric constant [8], features explained by Mie theory for the case of spherical particles [9].

While chemical reduction of metal salts is a common approach used to synthesize metallic nanoparticles, laser ablation was used effectively as an alternative method of synthesis $[1,10]$, consisting in the ablation of a target immersed in a liquid medium by means of a high power laser. The material generated is plasma containing electrons, ions and atoms from the target, confined by the medium high density. In this condition, electronic recombination occurs by rapid cooling causing the ions change to a neutral state favoring the formation of nanoparticles. In comparison to chemical reduction method, laser ablation allows a faster production of nanoparticles without the use of reagents that may contaminate their surface [11].

Synthesis of nanoparticles for a specific application requires considering stable suspension for a relative long period of time. To improve stability using laser ablation confined in a liquid medium, some studies propose the use of aqueous solutions of surfactants or polymers, but these additives can reduce the purity of the nanoparticles surface [12]. However, pure liquids such as distilled water, acetone, isopropyl alcohol, have been considered to favor the stability period without additional reagents $[11,13,14]$. This improvement in stability has been explained by the electric dipole moment and liquid viscosity [13].

Un like propanol, if methanol and ethanol are used for the synthesis, the agglomeration of nanoparticles and subsequent sedimentation occurs shortly $[11,13,15]$, with the drawback that these solvents decrease the transparency 
percentage with near-infrared light and are flammable. Certainly this can cause a fire, especially if in the experiment the $1064 \mathrm{~nm}$ emission of the Nd:YAG laser is focused from the top of the vessel while the liquid remains exposed to the environment. To cope with this problem, this work shows an experimental configuration that uses an Argon gas environment in the synthesis for laser ablation confined in methanol and ethanol, allowing to investigate the suspension stability of the silver nanoparticles produced in both solvents with this environment, and settling much safer operation conditions during the ablation process, especially when high fluence and fast ablation rate is performed.

\section{Experimental}

Figure 1 shows a scheme of the experimental setup used in nanoparticles preparation where top incidence is selected. This configuration uses the fundamental emission $(\lambda=1064 \mathrm{~nm})$ of a Nd:YAG pulsed laser, with an energy of 0.1 $\mathrm{J} /$ pulse and a repetition rate of $10 \mathrm{~Hz}$. Each synthesis takes an irradiation time of 10 minutes, adjusting the laser pulse diameter to a $1 \mathrm{~mm}$ by means of a lens with $20 \mathrm{~cm}$ focal length. We employed a silver coin of $99.99 \%$ purity as a target, cleaned with distilled water and ethanol to remove impurities. In addition, during the interaction with the laser, the target was kept in rotation with continuous movement controlled via software to reduce damage on the target surface. During synthesis the target was immersed in $12 \mathrm{ml}$ methanol or ethanol with $99.8 \%$ and $99.5 \%$ purity, respectively. A plastic cover is used to avoid the splashing to the surroundings outside of the vessel. At the same time, this cover allows to inject a flux of high purity Argon gas (99.997\%), with the aim of purging Oxygen from the vessel in order to prevent combustion ignition. The improvements in the experimental design has several advantages: first, the experiments can be run under more stable and safer conditions, since there is no possibility of combustion; and second, this configuration can be used in a more continuous or even industrial operation where higher laser energies and faster production rates are needed.

For the analysis, UV-Vis absorption spectra were obtained with a Perkin-Elmer 330 spectrophotometer. Silver nanoparticles were prepared on nickel grids for observation by Transmission Electronic Microscopy using a JEOLJEM-2010 and JEOLJEM-2100F with acceleration voltages of $150 \mathrm{kV}$ and $200 \mathrm{kV}$, respectively.

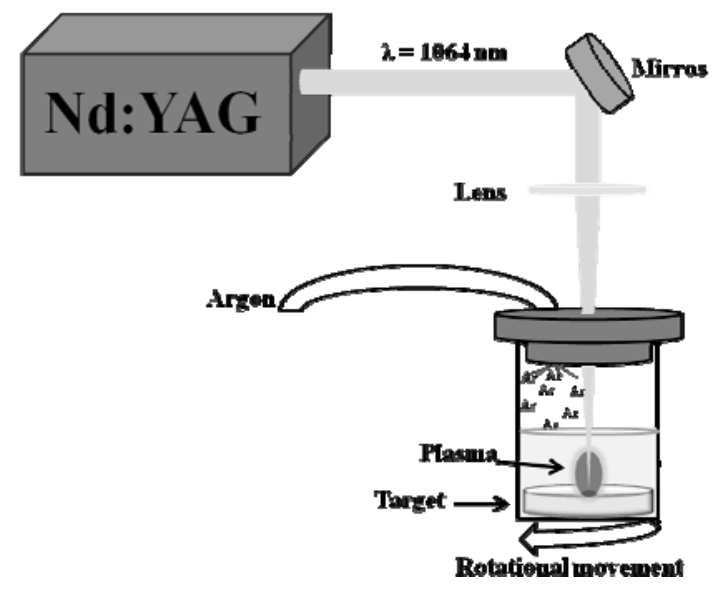

Fig. 1 Experimental configuration employed for the synthesis of silver nanoparticles by laser ablation confined in a liquid medium.

\section{Results and discussion}

First tests were performed in order to probe the instrumental setup. Effectively, without the introduction of Argon atmosphere combustion ignited most of the times. When Argon gas was injected to the vessel there was no combustion, even for higher values of laser energies and higher repetition rates. Once experimental conditions were established, synthesis begun, keeping the samples to room temperature. Little sediment was observed in the silver nanoparticles synthesized in methanol, showing a pale yellow color 1 month later. But in ethanol the nanoparticles settled in less than 24 hours showing a colorless liquid.

Figure 2 presents the histograms of size distributions of synthesized nanoparticles and the corresponding TEM images as insets. The number of particles used in these distributions was 177 and 232 particles for methanol and ethanol, respectively. Size distribution histograms were fitted with Lorentzian curves; one Lorentzian for the case of methanol and two for the case of ethanol. In the case of ethanol, the higher peak corresponds to nanoparticles with smaller sizes (centered around $14 \mathrm{~nm}$ ), while the center of the second peak is about the double (at $30 \mathrm{~nm}$ ). 

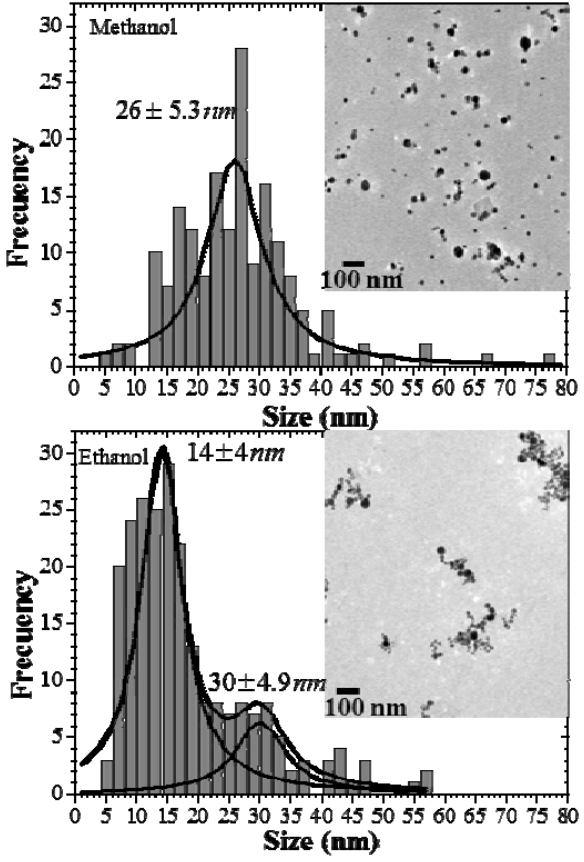

Fig. 2 Size distributions of silver nanoparticles prepared in methanol (up) and ethanol (down). And the respective transmission electron images are shown as insets.

The central position of each peak as well as their standard deviation is shown at the top of the same figure. Notice that in methanol the mode is larger than in ethanol and, at the same time, the nanoparticles prepared in methanol turned out to be dispersed (also shown in Figure 3 ). This can explain why there was no sediment observed in this sample, since it favored the stability of the nanoparticles in suspension with the environment. Whereas, in the TEM images obtained for nanoparticles synthesized in ethanol it is observed that the opposite happened, groups of aggregated nanoparticles were observed, explaining the rapid sedimentation; probably due to the ease of aggregation, the nanoparticles reached a critical mass to sediment.

It is important to remark that results obtained for nanoparticles synthesized in ethanol are consistent with results reported in the literature $[11,14,15]$, since they show images of aggregate silver nanoparticles and mention that in this solvent there was rapid sedimentation (about 48 hours). Nevertheless, in the case of methanol the results do not correspond to those previously reported in the literature, in which suspension inestability of nanoparticles synthesized in methanol behaves in the same way to

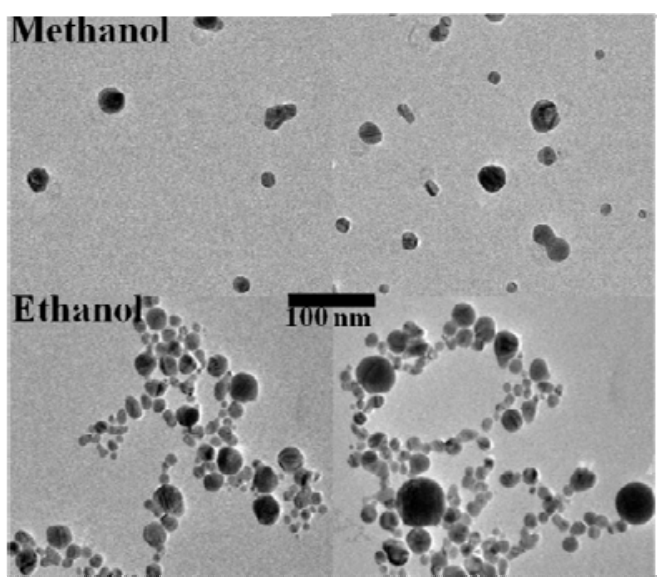

Fig. 3 Transmission electron micrographs with a higher magnification for nanoparticles obtained in methanol (upper micrographs) and ethanol (lower micrographs

those in ethanol. For example, similar experiments were reported in [11] and [16] were Ar gas is not used in the synthesis and, the main results show that silver nanoparticles produced with Nd:YAG pulsed laser with emission of 532 $\mathrm{nm}$ and the fundamental emission of $1064 \mathrm{~nm}$ exhibits the same instability suspension for ethanol and methanol. Effectively, Figure 4 recalls those results and clearly shows the agglomeration of nanoparticles leads to a same degree of sedimentation in both solvents.

By using the instrumental setup proposed in this work, silver nanoparticles synthesis in methanol exhibit more dispersion, resulting in better suspension stability. This difference in stability, with respect to other works, could be influenced by the injection of Argon gas, which in the beginning was used to prevent ignition of these flammable solvents. A possible explanation is that methanol admitted a larger quantity of argon ions, increasing the collision on groups of agglomerated nanoparticles, causing a major dispersion between them. This hypothesis arises from the fact that methanol and ethanol have different molecular number density, so that in methanol should cross a major quantity of argon ions, as can be showed in this relation $n=\rho / M \quad$ [\#molecules $/ \mathrm{cm}^{3}$ ], where $n, \rho$ and $M$ are the molecular number density, mass density and molecular mass of the molecules. Taking into account the values of $\rho$ and $M$ shown in Table, it is possible to write the ratio of numeric densities, such that $n_{\text {ethanol }} /$ $n_{\text {methanol }}=1.4$; which tells us that methanol has the smallest numerical density of molecules, being probable that this influenced a major argon ion collision of nanoparticle clusters. But 
to prove this hypothesis a more thorough investigation with other inert gases or different pressure levels of argon gas must be performed; certainly this is out of the scope of this work and is going to be the subject of a future research.
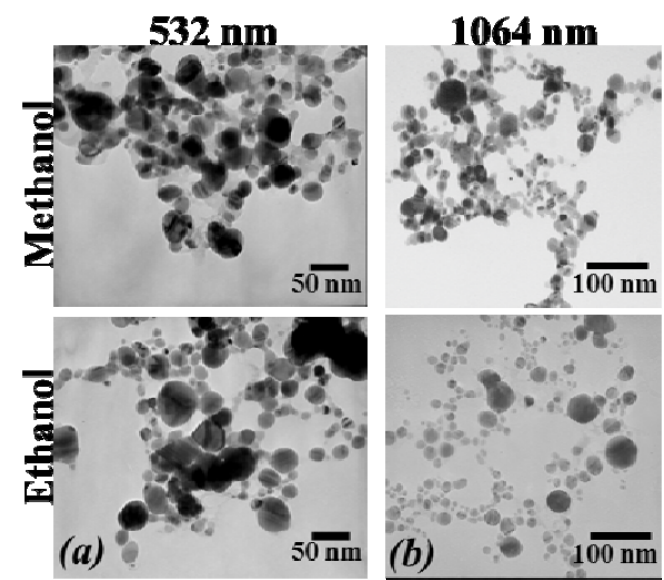

Fig. 4 (Left) Transmission electron micrographs nanoparticles obtained by laser emission at $532 \mathrm{~nm}$ [11]. (Right) micrographs of nanoparticles obtained by the fundamental emission [16].

Table 1 Mass density and molecular mass of methanol and ethanol

\begin{tabular}{|c|c|c|}
\hline Solvent & $\boldsymbol{\rho}\left(\mathbf{g} / \mathbf{c m}^{3}\right)$ & $\mathbf{M}(\mathbf{g} / \mathbf{m o l})$ \\
\hline Methanol & 0.79 & 32.04 \\
\hline & & \\
Ethanol & 0.78 & 46.07 \\
\hline
\end{tabular}

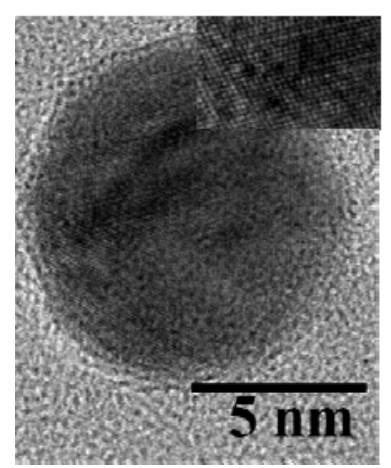

Fig. 5 High-resolution image of a nanoparticle obtained in methanol. A small area is shown as inset
High-resolution image of a single nanoparticle obtained in Methanol is shown in Figure 4, which exhibits an ordered atomic arrangement, indicating crystalline nature of these nanoparticles.

UV-Vis absorption spectra of nanoparticles obtained with the two solvents are shown in Figure 5. Both spectra present an absorption peak around $400 \mathrm{~nm}$, which is due to the surface plasmon resonance in silver. Nevertheless, the spectra profile is different, because the nanoparticles suspended in methanol show a well-defined peak, which in accordance with the Mie theory belongs to nanoparticles with spherical geometry. Regarding the geometry, there is some correlation with the images of nanoparticles synthesized in this solvent (see Figure 3) because nanoparticles exhibit quasispherical shapes. In ethanol, the peak is broader, such that the spectrum seems to be a superposition of two peaks. To this purpose, this absorption spectrum was fitted with two gaussian curves, the first peak $(395 \mathrm{~nm})$ having more intensity than the second one $(536 \mathrm{~nm})$, as can be seen in the figure. It is possible that agglomeration of a few nanoparticles gave this extended shape, taking into account the discrete dipole approximation (DDA), which also states that optical response depends on the shape of nanoparticles; where in this case a plasmon longitudinal mode contributed to light absorption in longer wavelengths [17]. Liao et al. reported [18] a similar result for a sample of gold nanoparticles in ethanol prepared by reduction of metallic salts, showing images of particle clusters and a broader absorption peak of appearance similar to those obtained in this work. Such agglomeration is explained with the dipole-dipole interaction model, which is favored by the charge differences in the surface of nanoparticles. Furthermore, the generalized Mie theory for linear chains of spherical nanoparticles presented the synthetic spectra of the extinction efficiency with a similar appearance, using silver refractive index [19]. Particularly, in the models the extinction efficiency is important by the correspondence they have with the absorbance, being useful to acquire better knowledge of the use of light to characterize nanomateriales. 


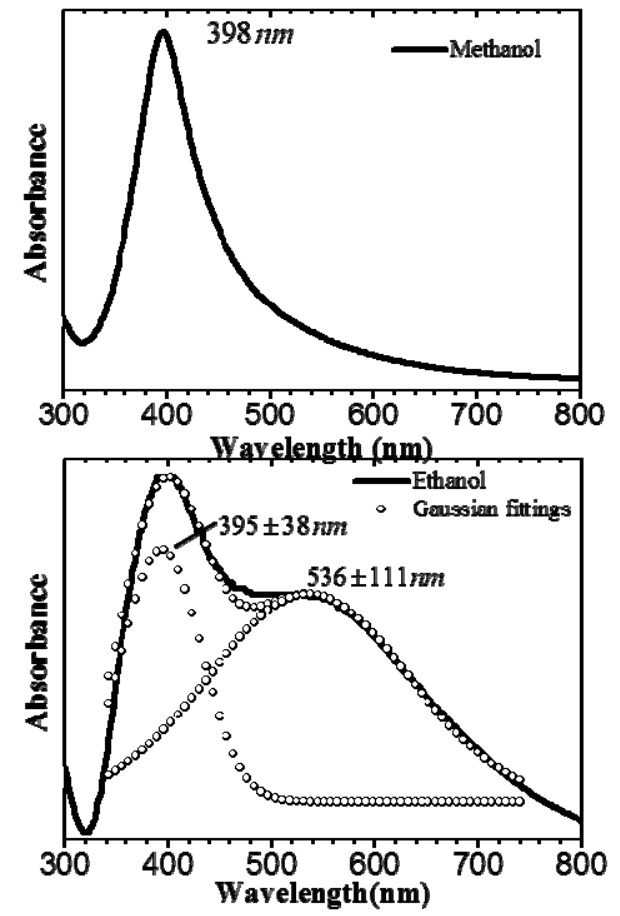

Fig. 6 UV-Vis absorbance spectra for nanoparticles prepared in methanol (up) and ethanol (down).

Figure 7 compares the size distribution of the nanoparticles obtained in methanol the day of the synthesis and after one month. Considering 229 particles after one month of the synthesis, the statistic shows narrower size distribution (from 5 to $44 \mathrm{~nm}$ ). In this case, the size distribution consist of two peaks where the first is smaller $(13 \mathrm{~nm})$ than the day of the synthesis (26 nm), but it is important to point out that in both images the nanoparticles appear dispersed, suggesting that in this solvent, certain amount of nanoparticles were suspended during this period, where little sediment was observed.

\section{Conclusions}

The experimental setup used in this work is important because Argon atmosphere helps preventing the combustion of the solvent in the present of oxygen. This setup is useful for the cases where the confining solutions favor the reactivity with oxygen, and when high fluence and fast repetition laser pulses are needed.
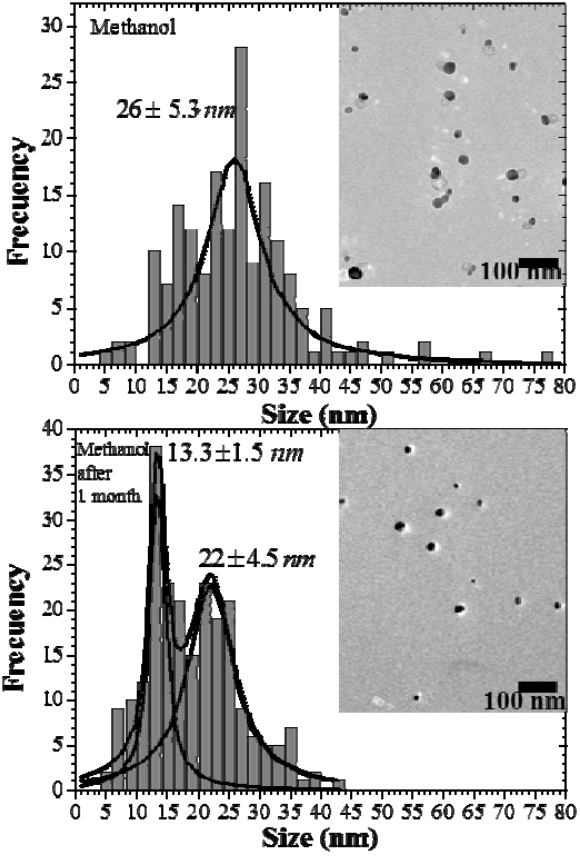

Fig. 7 Size distribution of silver nanoparticles prepared in methanol the day of synthesis (up) and after one month (down). Respective transmission electron images are shown as insets.

According to the results, the use of argon gas in laser ablation experiments confined in alcohols had an effect on the stability of the silver nanoparticles, mainly those prepared in methanol. The images of nanoparticles obtained in ethanol show strong agglomeration, leading to lower suspension stability; in accordance with the literature. On the contrary, nanoparticles synthesized in methanol were well dispersed, as shown in micrographs for the day of the synthesis and after one month.

\section{Acknowledgments}

Authors want to thanks the financial support from Consejo Nacional de Ciencia y Tecnología (CONACYT) and the Universidad NacionalAutónoma de México (UNAM) under program PAPIIT:IN105914. Also, we want to thank CNYN-UNAM for the space and facilities to realize the research. David Oseguera wants to thank to the Centro de Investigación Científica y de Educación Superior de Ensenada (CICESE) for the postdoctoral scholarship under CONACYT program. In addition, authors deeply thank the technical assistance of Francisco Ruiz Medina, Jaime Mendoza and Juan Velarde Magaña of the CNYN-UNAM. 


\section{References}

[1] J Neddersen, G Chumanov ,T.M Cotton , Appl. Spectrosc., 47, (1993) 1959

[2] L.P Ding, Y Fang, Appl Surf Sci., 253, (2007) 4450

[3] A.S Nikolov, N.N Nedyalkov, R.G Nikov, P.A Atanasov, M.T Alexandrov, D.B Karshanova, Appl Phys A., 109, (2012) 315

[4] G.M Herrera, A.C Padilla, S.P Hernández Rivera, Nanomaterials. 3, (2013) 158

[5] D Dorranian, S.A Afshar, N Tahmasebi, A.F Eskandari, J Clust Sci., 25, (2014) 1147

[6] M.M Kholoud, Abou El-Nour, Ala'aEftaiha, Abdulrhman Al-Warthan, Reda A.A. Ammar, Arabian Journal of Chemistry. 3, (2010) 135

[7] C Noguez, J. Phys. Chem. C. 111, (2007) 3806

[8] D.C Schinca, L.B Scaffardi, F.A Videla, G.A Torchia, P Moreno, L Roso, J ApplPhys D 42, (2009) 1

[9] C.F Borhen and D.R Huffman, Absorption and Scattering of Light by Small Particles (Wiley, New York, 1983)

[10] A Fojtik, A Henglein, Ber Buns. PhysChem 97 (1993) 252

[11] D. O Oseguera Galindo, A Martínez Benítez, A Chávez Chávez, G Gómez Rosas, A Pérez Centeno, M.A Santana Aranda, J Nanopart Res. 14, (2012) 1133

[12] F Mafuné, J. Y Kohno, Y Takeda . T Kondow, H Sawabe, J. Phys. Chem. B., 105, (2001) 5114

[13] J. S Jeon, C.H, Yeh, J Chin ChemSoc., 45 (1998) 721

[14] R. M Tilaki, A Irajizad, S. M Mahdavi Appl Phys A., 84 (2006) 215

[15] R. M Tilaki, A Irajizad, S.M Mahdavi, Appl Phys A., 88, (2007) 415

[16] D. O Oseguera Galindo, "Estudio de condiciones físicas y químicas en la síntesis de nanopartículas de plata por ablación láser confinada en medios líquidos", $P h D$ thesis , University of Guadalajara (2013)

[17] V Amendola, O.M Bakr, F Stellaci, Plasmonic., 5, (2010) 85

[18] J Liao, Y Zhang, W Yu, L Xu, C Ge, J Liu, N Gu, Colloid Surf A., 223, (2003) 177

[19]M Quiten, Optical Properties of Nanoparticles System: Mie and beyond (Wiley, New York, 2010) 